REVUE DE L'INSTITUT

FRANÇAIS D'HISTOIRE

EN ALLEMAGNE

\section{Revue de l'IFHA}

Revue de l'Institut français d'histoire en Allemagne

$2 \mid 2010$

IFHA 2

\title{
Le Verein für Reformationsgeschichte
}

\section{Marion Deschamp}

\section{OpenEdition}

\section{Journals}

Édition électronique

URL : http://journals.openedition.org/ifha/236

DOI : 10.4000/ifha.236

ISSN : 2198-8943

\section{Éditeur}

IFRA - Institut franco-allemand (sciences historiques et sociales)

\section{Édition imprimée}

Date de publication : 1 janvier 2010

Pagination : 103-106

ISSN : 2190-0078

\section{Référence électronique}

Marion Deschamp, « Le Verein für Reformationsgeschichte », Revue de I'IFHA [En ligne], 2 | 2010, mis en ligne le 01 février 2013, consulté le 19 avril 2019. URL : http://journals.openedition.org/ifha/236 ; DOI : $10.4000 /$ ifha.236

Ce document a été généré automatiquement le 19 avril 2019

(CIFHA 


\title{
Le Verein für Reformationsgeschichte
}

\author{
Marion Deschamp
}

1 Le 13 février 1883 est fondé, à Magdebourg, le Verein für Reformationsgeschichte. 125 ans plus tard, en 2008, le professeur d'histoire moderne Luise Schorn-Schütte en retrace, de manière courte mais saisissante, l'archéologie, l'histoire et les tribulations singulières, dans le 200ème volume des écrits (Schriften) publiés par l'association elle-même. Évitant de tomber de Charybde en Scylla, du panégyrique de commande à l'examen de conscience un brin tartufe, cette courte présentation historique fait plutôt entrevoir la fascinante puissance réflexive de son objet. Car le Verein ne s'est pas contenté, depuis sa création à l'époque wilhelminienne jusqu'à notre décennie, de refléter, de manière spéculaire, la transmutation de nombreuses sociétés de recherche allemandes, à prime vocation confessionnelle et militante, en institutions scientifiques sécularisées. Il ne s'est pas non plus satisfait d'acter scrupuleusement les symptômes de ces bouleversements sociétaux à chacun de ses frémissements. Il semble, aussi, avoir su impulser ses propres métamorphoses et surtout épingler, pour mieux les observer, les peaux de ses différentes mues, apportant ainsi son écot personnel aux thèses évolutionnistes de la recherche. Pourquoi donc ne pas nous pencher à notre tour, dans ce portrait, sur cette planche d'histoire?

2 En 1883, donc, naît le VRG, à l'initiative conjointe d'hommes d'Église et de professeurs des universités protestantes. Ceux-ci prennent appui sur le 400ème anniversaire de la naissance de Martin Luther pour afficher leur volonté de propager « l'identité protestante nationale ", et consolider la communauté luthérienne dans sa confiante conscience d'ellemême. Si l'élan virulent du Kulturkampf impulsé par Bismarck au début des années 1870 est déjà sur le point de s'essouffler politiquement (l'heure est aux négociations vaticanes), l'offensive confessionnelle contre les catholiques allemands, stigmatisés comme ennemis de l'intérieur, n'en semble que plus forte, une fois circonscrite dans l'orbe des institutions intellectuelles et des cénacles académiques. Dans ce contexte particulier, le VRG ne cache pas son ambition de participer à ce militantisme confessionnel, par la promotion de l'histoire de la Réformation interprétée comme élément fondateur de l'identité nationale. Très vite, il développe les instruments appropriés à la défense de sa cause : une collection d'ouvrages (Schriftenreihe), conçue pour ériger autant de monuments littéraires au 
Réformateur, et servir de contre-offensive aux publications catholiques (d'un Janssen, par exemple, ou d'un Denifle) esquintant, elles, sans ménagement, la figure hérö̈que de Luther ; et une revue, l'Archiv für Reformationsgeschichte (ARG), qui paraît dès 1903 mais ne tombe sous la responsabilité éditoriale du Verein qu'à partir de 1920.

Si l'anticatholicisme politico-confessionnel du VRG se maintient plus longtemps que l'Empire wilhelminien et tout au long de la République de Weimar, il doit composer à partir des années 1920 avec une tendance à la « scientifisation " (Verwissenschaftlichung) des activités de l'association. Répondant autant à une crise financière qu'à un processus d'institutionnalisation des sociétés savantes dans le monde de la recherche allemande, les travaux du Verein se professionnalisent en abandonnant peu à peu les oripeaux partisans et militants de ses origines. La compromission de son président Otto Scheel avec le régime national-socialiste, qui explique en partie que le Verein n'ait pas cessé d'exister pendant la Seconde Guerre mondiale, n'entame pas, au contraire, ce processus de déconfessionnalisation (Entkonfessionalisierung), ni l'accentuation du caractère scientifique des recherches menées par le VRG. Dès 1938, en fait, celui-ci opère un élargissement de son programme de recherche, dans lequel le strict intérêt pour l'histoire ecclésiologique de la Réforme allemande et pour la théologie luthérienne se craquelle au profit d'autres zones géographiques, et d'autres confessions chrétiennes. Après-guerre, sous l'influence déterminante d'Heinrich Bornkamm, le Verein accélère encore ce mouvement d'ouverture. Ouverture orientée, cependant, par un double tropisme occidental: le déplacement du siège du VRG de Magdebourg à Heidelberg, d'une part, entant l'institution sur le sol ouest-allemand; et, d'autre part, la collaboration transatlantique, jusqu'à aujourd'hui encore non démentie, établie avec des partenaires de recherche américains. Dès 1951, ainsi, l'ARG devient une revue internationale et bilingue, publiée conjointement par le VRG et la Society for Reformation Research.

Depuis 1973 et la définition des nouveaux statuts du VRG, qui en font une association à but non lucratif, celle-ci s'est explicitement coupée de sa base luthéro-confessionnelle tout en adoptant cette maxime trinitaire annoncée comme une nouvelle antienne: Interdisziplinarität, Überkonfessionalität, Internationalität. Les auteurs publiés par le VRG, sont en effet autant des historiens de l'Église (Kirchenhistoriker) que des historiens " généralistes ", mais aussi des anthropologues, des historiens d'art, ou de la littérature. L'engagement à faire une histoire trans- ou supraconfessionnelle de la Réformation est quant à lui bien la trace d'une sécularisation de la recherche, et non, comme c'était peutêtre le cas après-guerre, d'une tendance œcuménique traversant autant les Églises que la société toute entière. Ce phénomène est par exemple visible à travers le cas des Schriften de la VRG consacrées aux confessions " hétérodoxes » issues de la fameuse "aile gauche de la Réformation ». Si les premiers travaux traitant de l'anabaptisme ou d'autres groupes religieux dissidents s'y intéressaient moins en tant que sectes au sens troeltschien qu'en Églises déviantes par rapport à l'orthodoxie luthérienne, cette dernière (elle-même remise en cause en tant que fondement stable) a cessé d'être systématiquement considérée comme l'étalon de mesure idéal-typique des autres groupes confessionnels. De même, à partir des années 1970, la connaissance et l'investigation des deux autres grandes confessions chrétiennes, réformée et catholique, ont été entreprises sur la base d'une égalité affichée et d'un parallélisme assumé avec leur consœur luthérienne. En 1993, l'organisation collégiale, avec la Société catholique pour l'établissement du Corpus Catholicus (katholische Gesellschaft zur Herausgabe des CC), d'un colloque portant sur la confessionnalisation catholique a été l'une des manifestations concrètes de ces nouvelles 
exigences. Enfin, plus récemment, le rôle de l'Islam face à la Réformation a fait son entrée dans les terrains d'études renouvelées du VRG.

5 Mais, comme nous l'écrivions en commençant ce portrait, le Verein ne se contente pas de refléter les évolutions du « fait » confessionnel dans l'organisation des savoirs historiques modernes. En prenant pour objet l'histoire de la Réformation et de ses conséquences, c'est-à-dire précisément l'émergence du temps confessionnel (konfessionelles Zeitalter), certes liquidé par les traités de 1648 mais dont le Kulturkampf bismarckien est une reviviscence, le Verein aura joué un rôle actif dans la définition même du fait confessionnel européen, et dans la compréhension de ses prémices. Les travaux majeurs lancés à la fin de la décennie 1970 et au cours de la décennie suivante, autour du concept de Konfessionalisierung, érigé par l'historien Heinz Schilling à la dignité de paradigme interprétatif, en administrent la preuve. Depuis, le VRG ne cesse, par le truchement de l'ARG ou des Schriften qu'il publie, et par la prise en compte de la diversité des positions historiographiques, de réinterroger ses propres acquis historiques et notamment la validité des paradigmes qu'il a lui-même contribué à forger (voir, par exemple, le volume publié en 2008 sur les Konfessionskulturen). Il s'avère en ce sens une véritable fabrique notionnelle sur le confessionnel.

Aujourd'hui, le VRG compte près de 400 membres, dont à peu près deux tiers de personnes privées et le tiers restant d'institutions variées (bibliothèques, instituts de recherches, institutions religieuses). Il est administré par un comité directeur composé de professeurs d'université (historiens et historiens de l'église) de cinq nationalités (allemande, en majorité, mais aussi suisse, américaine, française et polonaise) et présidé par le professeur d'histoire moderne Heinz Schilling (Humboldt-Universität, Berlin). L'association participe activement au soutien de la recherche en faisant paraître, outre sa Schriftenreihe qui comporte actuellement plus de 200 titres, et l'ARG qui est devenue quadrilingue (intégrant des communications en français et en italien), une collection de sources publiées. Enfin, elle organise journées d'études et colloques qui exercent un véritable patronage (laïc) sur le monde de la recherche historique de la Réformation. Cette omniprésence et cette effervescence pourraient presque être jugées pesantes si le VRG ne s'efforçait continuellement d'intégrer, sans l'annuler, la diversité des historiographies, parfois contradictoires, qui émaillent le terrain jamais épuisé de l'histoire de la Réformation et de l'ère confessionnelle.

\section{AUTEUR}

\section{MARION DESCHAMP}

Marion Deschamp est boursière d'aide à la mobilité à l'IFHA. 